\section{Design and Applications of Nanoparticles in Biomedical Imaging}

J.W.M. Bulte and M.M.J. Modo, Eds.

New York, NY: Springer, 2017, 468 pages, $\$ 239$

Imaging and therapeutic delivery are increasingly relying on nanoparticles as a key technology platform. This common technology interface affords new opportunities to combine diagnosis and therapy into a unified 1-step theranostic approach. Because of the varied chemical compositions of nanoparticles, they are now emerging as a main driver of innovation in the development of novel imaging applications, especially in nanomedicine.

This book, written by 86 contributors and organized into 21 chapters, covers the most recent advances in using nanoparticles for biomedical imaging, including MRI, magnetic particle imaging, nuclear medicine, ultrasound imaging, CT, and optical imaging. Topics include nanoparticles for MRI and magnetic particle imaging, small interfering RNA delivery, theranostic nanoparticles for PET of drug delivery, ultrasound nanoparticles for imaging drug delivery, inorganic nanoparticles for targeted CT, and quantum dots for optical (fluorescent) imaging. World-leading experts describe the chemistry, imaging acquisition, and image processing, as well as the requirements for clinical translation. Applications range from cell tracking in regenerative medicine to early detection of cancer and inflammation across all major imaging modalities. Most figures are in color and clear, and the tables are informative. The references are up to date, and the index is helpful.

This book is a valuable resource on the fundamental science of diagnostic nanoparticles and interactions with biologic targets, providing a practical guide to improved detection of diseases and its clinical implementation. This timely book also provides a basis for further innovation and synergy in the fields of nanomedicine. I highly recommend this book to academic physicians, scientists, and trainees in diagnostic imaging and nuclear medicine.

\author{
E. Edmund Kim \\ University of California at Irvine \\ 101 The City Dr. S. \\ Orange, CA 92868 \\ E-mail: edmundek@uci.edu
}

Published online Oct. 6, 2017

DOI: $10.2967 /$ jnumed.117.202358

\title{
Erratum
}

In the article "Synthesis of ${ }^{18} \mathrm{~F}$-Tetrafluoroborate via Radiofluorination of Boron Trifluoride and Evaluation in a Murine C6-Glioma Tumor Model" by Jiang et al. ( Nucl Med. 2016;57:1454-1459), two grant acknowledgments were inadvertently left out of the article. The following two grants should have been included: R01 CA196975/CA/ NCI NIH HHS/United States and R21 HL127389/HL/NHLBI NIH HHS/United States. The authors regret the error. 